\title{
La materialidad en auditoría como barrera hacia la comparabilidad de la información financiera Una revisión de la investigación empírica previa
}

\author{
Francisco Javier Martínez García* \\ Ana Fernández Laviada ** \\ Javier Montoya del Corte $* * *$
}

\section{Resumen}

Iniciativas como las Normas Internacionales de Contabilidad e Información Financiera (NIC/NIIF), asi como las Normas Internacionales de Auditoría (NIA) suponen un gran impulso hacia la armonización de la información corporativa. No obstante, en su proceso de implantación encontramos ciertas imprecisiones que vienen existiendo desde hace décadas; una de ellas es la aplicación del concepto de materialidad en contabilidad y auditoría. El objetivo de este trabajo es demostrar, a través de una revisión de la bibliografia previa especializada, que la ausencia de pautas obligatorias para determinar la materialidad en los trabajos de auditoría origina una continua falta de consenso entre los individuos, lo cual desfavorece el proceso de establecimiento de sistemas que permitan la comparabilidad plena de la información financiera publicada por las empresas.

Palabras clave: materialidad, armonización, comparabilidad, formación de juicios.

Fecha de recepción: 15-02-2006

Fecha de aceptación: 11-01-2007

\section{Materiality in Auditing as a Barrier to Financial Reporting Comparability: a Review of the Literature}

\begin{abstract}
The harmonization of the corporate financial reporting is driven by initiatives such as International Accounting and Financial Reporting Standards (IAS/IFRS) and International Standards on Auditing (ISA).

\footnotetext{
* Profesor investigador de la Facultad de Ciencias Económicas y Empresariales de la Universidad de Cantabria. Correo electrónico: martinfj@unican.es

** Profesora investigadora de la Facultad de Ciencias Económicas y Empresariales de la Universidad de Cantabria. Correo electrónico: fernanda@unican.es

***Profesor investigador de la Facultad de Ciencias Económicas y Empresariales de la Universidad de Cantabria. Correo electrónico: montoyaj@unican.es
}

No. 222, mayo-agosto 2007:21-40 
However, the implementation of the harmonization process is hampered by a lack of definitional clarity arising, for example, from the application of the materiality concept in accounting and auditing. The aim of this paper is to show by reviewing the relevant empirical literature that the lack of mandatory guidelines with regard to materiality allows the existence of an ambiguity among auditors and other groups (i.e. producers and users of financial reports). This militates against a full comparability of the financial information disclosed by companies.

Key words: Materiality, Harmonization, Comparability, Judgments.

\section{Introducción}

$\mathrm{C}$ Tomo señala el "Marco Conceptual para la preparación y presentación de estados financieros" (IASB, 1989), éstos tienen como objetivo suministrar información útil a diversos usuarios sobre la situación financiera, los resultados y los flujos de efectivo de las entidades. Dos de los organismos profesionales que más intensamente trabajan en la actualidad en favor de disponer de unos estados financieros más comprensibles, relevantes, fiables y comparables son el International Accounting Standards Board (IASB) — a través de la emisión de Normas Internacionales de Contabilidad e Información Financiera (NIC/NIIF) - y el International Auditing and Assurance Standards Board (IAASB) de la International Federation of Accountants (IFAC) — mediante la elaboración de Normas Internacionales de Auditoría (NIA).

Las NIC/NIIF son un instrumento de armonización contable internacional que favorecen la comparabilidad de la información financiera; por su parte, las NIA representan un esfuerzo por establecer unas normas de auditoría de elevada calidad con el propósito de que esta actividad se desarrolle en el ámbito internacional bajo unos estándares uniformes y homogéneos.

Sin embargo, en el proceso de armonización global de la contabilidad y la auditoría existen lagunas importantes que no han logrado salvarse con el paso de los años. Éste es el caso de la aplicación del concepto de materialidad, tanto desde el punto de vista de quien formula los estados financieros como de quien se encarga de su revisión y verificación. La ausencia de criterios definidos con carácter obligatorio y la importancia decisiva del juicio profesional de los individuos en la determinación de los niveles de materialidad van en detrimento de una información financiera plenamente comparable.

El objetivo de este trabajo es constatar, por medio de la revisión de la bibliografía especializada, que la falta de pautas preceptivas sobre materialidad puede perjudicar la comparabilidad de los estados financieros. Para ello, estructuramos el artículo de la siguiente forma: tras esta breve introducción, en el segundo apartado realizamos una aproximación al concepto de materialidad con el propósito de destacar su trascendencia y la problemática de su aplicación, y llevamos a cabo la delimitación de la revisión 
La materialidad en auditoría como barrera hacia la comparabilidad de la información financiera

Una revisión de la investigación empírica previa

efectuada; en los apartados tercero y cuarto, respectivamente, analizamos con detalle los resultados obtenidos por la investigación empírica previa, reveladores de la existencia de diferencias individuales significativas entre los profesionales de una de las disciplinas sujetas al concepto de materialidad como es la auditoría, así como entre éstos y los sujetos de otros colectivos - como contables, gestores de inversiones, analistas y ejecutivos financieros, prestamistas, estudiantes universitarios, entre otros- en relación con el proceso seguido para la formación de los juicios sobre materialidad; por último, en el quinto apartado presentamos las conclusiones que se desprenden de nuestro trabajo.

\section{Trascendencia y problemática del concepto de materialidad. Delimitación de la revisión}

El concepto de materialidad juega un papel esencial, e incluso decisivo, dentro del proceso seguido hasta la publicación de los estados financieros.

La NIC 1 dispone que todas aquellas partidas con la suficiente importancia o materialidad deben presentarse de manera separada en los estados financieros; en sentido opuesto, aquellas que no son significativas deben aparecer agrupadas con otras de similar naturaleza, ya sea en los estados principales o en las notas a los mismos. Además, el requisito de materialidad implica que no es necesario cumplir lo dispuesto en las NIC sobre presentación cuando la información correspondiente resulte poco significativa.

Por otra parte, la determinación de la materialidad influye notablemente en cualquier trabajo de auditoría de cuentas de la siguiente manera:

1) En la etapa preliminar de planificación se utiliza para concretar la naturaleza, alcance y momento de aplicación de los procedimientos de auditoría.

2) En la etapa de ejecución del trabajo, el fraccionamiento de la materialidad fijada en la fase de planificación da lugar al error tolerable, que representa el límite máximo de aceptación de errores con el que el auditor pueda concluir que el resultado de la prueba logró su objetivo de auditoría y se utiliza para determinar el alcance de las pruebas de auditoría en las diferentes áreas de trabajo.

3) En la etapa final de emisión del informe se utiliza como referencia para evaluar la significatividad de los errores detectados durante el trabajo y su inclusión en el dictamen del auditor.

En consecuencia, la determinación de las cifras de materialidad influye en la preparación y presentación de los estados financieros y de ellas depende el tipo de opinión emitida por el auditor.

$\mathrm{Al}$ restringir nuestro estudio a la aplicación de la materialidad en la auditoría, resaltamos que, a pesar de su trascendencia, éste es un concepto cuya concreción se efectúa bajo una problemática especial que resumimos en los siguientes tres puntos: 
1) No existen criterios o pautas cuantitativas de obligado cumplimiento para la fijación de los niveles de materialidad. En algunos países como España y México, los auditores únicamente disponen de parámetros orientativos para su determinación.

2) La información financiera, además, puede ser significativa por motivos tanto de cuantía como de naturaleza, de tal forma que partidas por importes reducidos pueden ser relevantes únicamente por su naturaleza (fraudes, incumplimiento de contratos, etc.).

3) La determinación de la materialidad recae en última instancia sobre el juicio profesional del auditor.

Por tanto, la ausencia de criterios obligatorios y la supeditación de las decisiones de materialidad al juicio profesional de los individuos han acarreado a lo largo de las décadas un comportamiento excesivamente heterogéneo entre los sujetos al formular sus juicios sobre materialidad. Dada la enorme relevancia de la cuestión, el IAASB hizo público en diciembre de 2004 el borrador de la NIA 320, La materialidad en auditoría, bajo el nuevo título NIA 320 (revisada), La materialidad en la identificación y evaluación de errores $^{l}$; en este borrador y en sus desarrollos posteriores, aunque se producen avances muy importantes, no se introducen pautas cuantitativas preceptivas y el juicio profesional de los auditores sigue jugando un papel primordial en las decisiones de materialidad.

Algunos estudios han revelado la existencia de diferencias individuales entre los auditores, mientras que otros han localizado discrepancias entre éstos y otros grupos de sujetos, contables y usuarios principalmente. Así, los resultados de determinadas investigaciones empíricas han puesto de manifiesto que esta discordancia entre los individuos puede estar motivada por diversas causas, entre las que destacamos las siguientes: 1) la consideración de distintos factores para establecer los umbrales de materialidad, tanto en número como en naturaleza, 2) una ponderación diferenciada de la importancia de estos factores y 3) la utilización de diferentes umbrales de materialidad.

Consecuentemente, esta falta de definición de criterios obligatorios hace que existan diferencias significativas en los procesos seguidos por los auditores y otros individuos para la formación de sus juicios sobre materialidad. De esta manera, la aplicación del concepto en la auditoría de cuentas influye negativamente en la comparabilidad de la información financiera emitida por las empresas.

En la tabla 1, presentamos los trabajos que hemos seleccionado y que constituyen el objeto de nuestra investigación, los cuales clasificamos en función de los criterios que establecimos para realizar nuestro análisis y exponer los resultados: 1) el grupo o grupos de sujetos donde se localiza la divergencia de juicios sobre materialidad y 2) la fuente principal de origen de la falta de consenso entre estos individuos.

\footnotetext{
1 A la fecha de revisión de nuestro trabajo (9 de enero de 2007) la NIA 320 seguía aún en proceso de revisión, si bien la misma ha sido dividida en dos normas diferenciadas, la NIA 320 (revisada y reeditada) y la NIA 450 (reeditada), ambas redactadas bajo el nuevo formato de claridad. Puede ampliarse esta información en la página web de la IFAC: http://www.ifac.org/IAASB/ExposureDrafts.php
}

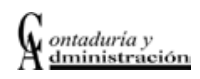


La materialidad en auditoría como barrera hacia la comparabilidad de la información financiera Una revisión de la investigación empírica previa

Tabla 1

Ausencia de consenso en los juicios sobre materialidad

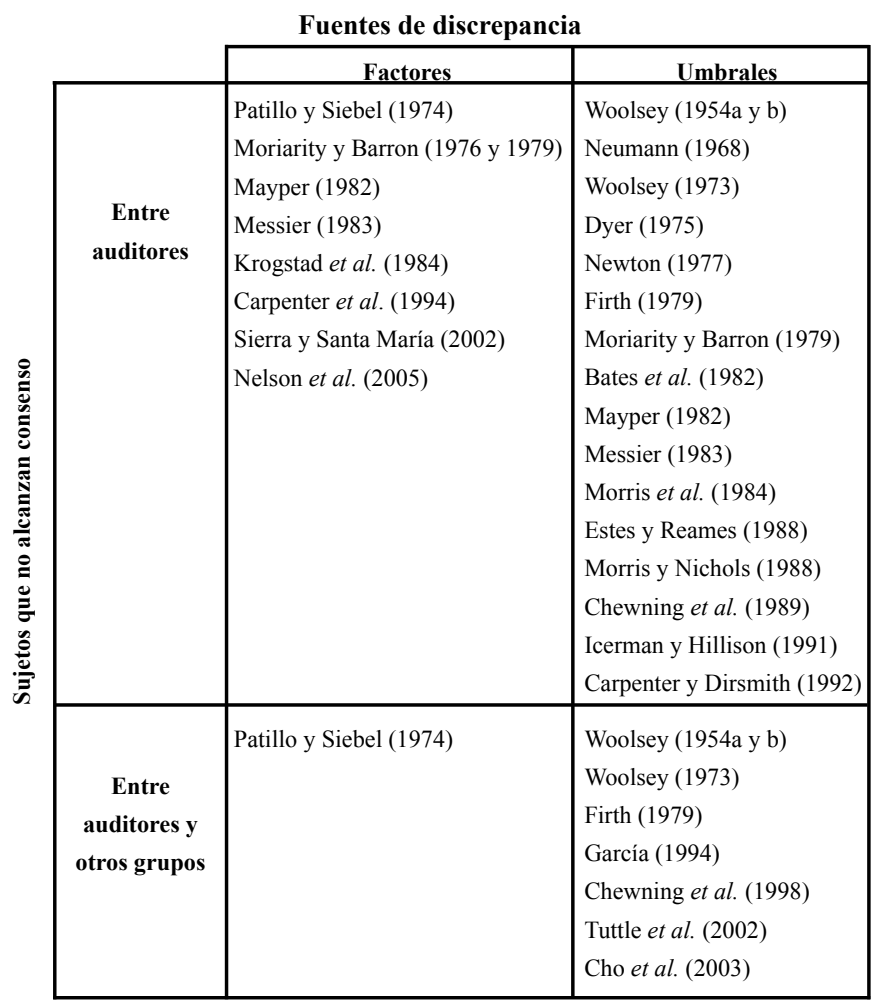

Fuente: Elaboración propia

Por razones de espacio, centramos fundamentalmente nuestra atención en los trabajos que muestran la ausencia de consenso entre auditores, limitándonos a presentar en el cuarto apartado un compendio a modo de tabla con los trabajos que identifican diferencias con relación a la materialidad entre auditores y otros grupos profesionales.

\section{Diferencias entre los auditores en la formación de los juicios sobre materialidad}

En el presente apartado, mostramos los resultados de aquellas investigaciones que han evidenciado que la carencia de pautas obligatorias sobre materialidad conlleva una falta de consenso entre los profesionales de la auditoría de cuentas al momento de formular este tipo de juicios.

\subsection{Diferencias en los factores considerados}

La observación de distintas variables de referencia o la ponderación desigual de la importancia de un mismo conjunto de factores orientativos han sido identificadas por muchas investigaciones 
Francisco Javier Martínez García, Ana Fernández Laviada y Javier Montoya del Corte

como causas de la ausencia de consenso entre los auditores en relación con sus juicios sobre materialidad. En la tabla 2, sintetizamos los resultados más destacados en este sentido.

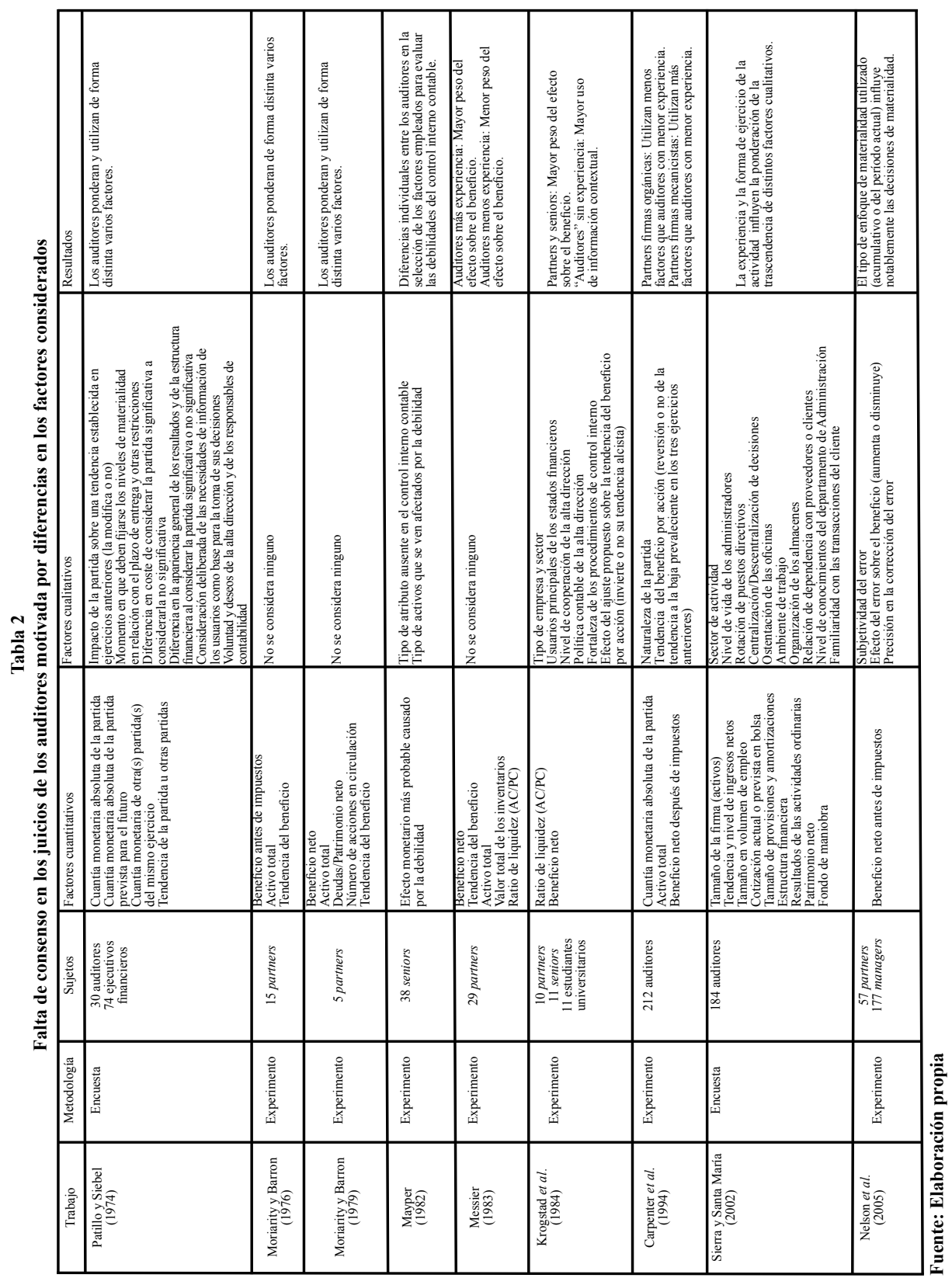


La materialidad en auditoría como barrera hacia la comparabilidad de la información financiera

Una revisión de la investigación empírica previa

Partimos en nuestra revisión del trabajo llevado a cabo por Patillo y Siebel (1974), quienes por medio de una encuesta tratan de conocer los factores empleados en la formación de los juicios sobre materialidad vinculados con determinadas áreas contables. Aunque en el análisis de resultados no discriminan entre auditores y ejecutivos financieros, puede deducirse la existencia de diferencias individuales dentro de los dos grupos en cuanto a la importancia que atribuyen a diez factores de naturaleza cuantitativa y cualitativa. En este sentido, y con carácter general, los auditores consideran que en la formación de los juicios sobre materialidad intervienen varios factores igualmente importantes. Sin embargo, hay individuos que no tienen en cuenta algunos de esos factores, otros piensan que los mismos tienen una importancia secundaria, mientras que un número reducido de participantes considera alguno de ellos como el único factor importante en los juicios sobre materialidad.

Igualmente, Moriarity y Barron (1976) demuestran que los auditores ponderan de forma diferente la importancia de dos factores de naturaleza cuantitativa cuando forman sus juicios acerca de la significatividad de una sobrestimación del beneficio por la consideración errónea de la vida útil de unos activos. La mayoría de los individuos que participan en su experimento poseen un modelo de decisión perfectamente aditivo (ocho de quince) o casi aditivo (tres de quince), de tal manera que sus decisiones de materialidad se corresponden con la suma de los efectos ponderados de la partida evaluada sobre las tres variables financieras consideradas: beneficio neto, activo total y tendencia del beneficio. Si bien para todos ellos el efecto de la partida sobre el beneficio neto ${ }^{2}$ es el factor dominante en sus decisiones, no están de acuerdo respecto a la trascendencia de los otros dos factores. Para algunos la tendencia del beneficio es la segunda variable más importante, mientras que para otros lo es el tamaño de la empresa, representado por el activo total.

Poco tiempo después, estos autores llegan a la misma conclusión con respecto a la determinación de las cifras de materialidad en la etapa de planificación de la auditoría. Del análisis de resultados efectuado por Moriarity y Barron (1979) se deriva una falta de consenso entre los auditores que participan en el experimento y la imposibilidad de establecer una representación común de sus procesos de formación de este tipo de juicios. A pesar de que para la mayoría el beneficio neto vuelve a ser el factor individual que más contribuye en la formación de los juicios sobre materialidad, para uno de los cinco auditores esta variable es menos importante que la cuantía total de los activos. Esta desavenencia también se muestra respecto al factor que representa la tendencia del beneficio, ya que afecta de forma desigual a los juicios sobre materialidad de los individuos. En consecuencia, los auditores participantes manifiestan un comportamiento distinto en el proceso de formación de sus juicios sobre materialidad porque otorgan diferente trascendencia a las variables consideradas, emplean más o menos factores como referencia y, como veremos más adelante, utilizan niveles de materialidad también diferenciados.

\footnotetext{
2 Una gran parte de los trabajos revisados destacan alguna medida del beneficio empresarial como el factor de referencia más importante en la formación de los juicios sobre materialidad: Véanse Woolsey (1954a y b, 1973), Dyer (1975), Moriarity y Barron (1976 y 1979), Firth (1979), Bates et al. (1982), Messier (1983), Krogstad et al. (1984), Chewning et al. (1989), Carpenter y Dirsmith (1992), Carpenter et al. (1994) y García (1994).
} 
Por otro lado, Mayper (1982) obtiene evidencia sobre la existencia de diferencias individuales entre los auditores cuando seleccionan los factores utilizados para ordenar según su importancia las debilidades en el control interno contable detectadas, concretados en el tipo de activo que se ve afectado por la debilidad, el tipo de atributo ausente en el control interno y el efecto monetario más probable originado por aquella. Adicionalmente, aunque constata un grado de consenso moderado en términos globales entre los participantes respecto a la significatividad de diferentes debilidades en el control interno contable, este nivel de acuerdo varía en función de la debilidad de que se trate.

Messier (1983), por su parte, utiliza la experiencia profesional como criterio de clasificación de los auditores y evalúa su influencia en la calidad de los juicios sobre materialidad. ${ }^{3}$ Utilizando como rubro para el análisis la depreciación del valor de unas existencias, verifica que los sujetos más experimentados hacen depender en mayor medida sus juicios sobre materialidad de un solo factor: el efecto del ajuste en el rubro sobre el beneficio neto. Contrariamente, los auditores con menor experiencia tienden a centrarse menos en esta variable y atienden a un mayor número de factores como base para la toma de sus decisiones de materialidad.

Unos resultados consistentes con este trabajo son obtenidos por Krogstad et al. (1984), quienes analizan el papel que juegan la experiencia y la información contextual en la formación de los juicios sobre materialidad de dos grupos de auditores y otro de estudiantes universitarios de contabilidad y auditoría. Sobre la base de ocho variables cuantitativas y cualitativas de referencia, los participantes deben evaluar la significatividad de una provisión para cuentas dudosas. Como conclusiones a su investigación señalan que, a medida que los auditores adquieren experiencia en su trabajo, la cantidad de información importante utilizada en la formación de los juicios sobre materialidad aumenta (aunque las diferencias no son significativas) y que los estudiantes son el grupo que mayor proporción de información contextual significativa incorpora y que menos se basa en el efecto de la partida sobre el beneficio neto para formar sus juicios. En este sentido, parece que los profesionales (partners y seniors) forman sus juicios sobre materialidad, principalmente, con base en la relación de la partida con el beneficio neto, ajustándolos en atención a distintas variables de naturaleza contextual (no financiera). Finalmente, aunque las diferencias no son significativas, el nivel de consenso y uniformidad en los juicios sobre materialidad de los partners es ligeramente superior que el de los seniors; sin embargo, estas diferencias sí son significativas si se unen los dos grupos de profesionales y se comparan con los estudiantes universitarios, pues los primeros son los que alcanzan unos juicios más consensuados y estables.

A través de otro experimento, Carpenter et al. (1994) advierten que la existencia de un comportamiento diferente entre los auditores en el proceso de formación de los juicios

\footnotetext{
${ }^{3}$ La calidad de los juicios sobre materialidad la mide atendiendo a cuatro criterios diferentes: grado de consenso alcanzado, ponderación de los factores utilizados, capacidad de auto-comprensión del proceso a seguir en su formación y estabilidad.
} 
La materialidad en auditoría como barrera hacia la comparabilidad de la información financiera

Una revisión de la investigación empírica previa

sobre materialidad respecto a distintas clases de cancelación anticipada de deudas es función simultánea de su experiencia y de la cultura de la firma a la que pertenecen. Al respecto, los autores exponen que a medida que los individuos incrementan su experiencia dentro de una firma de auditoría van incorporando de forma progresiva la cultura de la misma a su proceso de formación de juicios. Así, son los partners (mayor experiencia en comparación con seniors y managers) los que mejor reflejan la filosofía de su firma en relación con los juicios sobre materialidad. En primer lugar, dentro de las firmas orgánicas, donde los individuos tienen mayor autonomía y capacidad de maniobra, esta categoría de auditores se basa en el efecto sobre el beneficio neto y en su juicio profesional para tomar las decisiones de materialidad; por el contrario, en las firmas mecanicistas, donde los auditores están sometidos a un cumplimiento más estricto de los procedimientos establecidos, los partners incorporan una mayor cantidad de información al formar sus juicios sobre materialidad con base en un modelo establecido.

Sierra y Santa María (2002) llevan a cabo una investigación con el objetivo de conocer la relación entre las características individuales del auditor y los factores que prevalecen en la formación de sus juicios sobre materialidad en la etapa de planificación. Si bien no encuentran ninguna relación significativa entre el perfil de personalidad del auditor y los factores cuantitativos y cualitativos que éste utiliza en la determinación de los umbrales de materialidad, sí se producen relaciones significativas entre estos últimos y ciertas características profesionales. Así, observan que los auditores con mayor experiencia conceden mayor importancia al factor nivel de conocimientos del departamento de Administración en la formación de sus juicios sobre materialidad. En este mismo sentido, los auditores independientes valoran como importantes la centralización o descentralización de decisiones y el nivel de conocimientos del departamento de Administración, por lo que estos factores son considerados como poco importantes por los auditores que ejercen su actividad por cuenta ajena. Estos últimos, finalmente, también estiman como poco importante el factor que alude a la rotación de los puestos directivos.

Por último, Nelson et al. (2005) desarrollan un experimento con el propósito de analizar si el planteamiento usado como base para la toma de decisiones de materialidad afecta a la propuesta de ajustes por parte de los auditores y, por tanto, a la cantidad de errores incluidos en los estados financieros auditados. Al respecto, se describen dos enfoques utilizados en la práctica auditora en relación con el proceso de formación de juicios sobre materialidad, los cuales pueden hacer que la evaluación sea cuantitativamente más o menos significativa en función de la relación entre el número total de errores y los errores correspondientes al período actual. El primero de estos enfoques, acumulativo, compara con el beneficio neto la cantidad total de errores existentes al final del ejercicio actual; el segundo, del período actual, compara con el beneficio neto la cantidad de nuevos errores agregados en el ejercicio vigente. Los resultados de su investigación muestran la existencia de un efecto robusto del enfoque de materialidad utilizado sobre la proposición de ajustes por parte del auditor. De este modo, concluyen que los auditores son más propensos a requerir ajustes bajo el enfoque de auditoría que hace parecer al error 
cuantitativamente más importante. Este resultado se mantiene con independencia de la magnitud del error, su subjetividad, su impacto sobre el beneficio, la opinión del auditor sobre si el enfoque empleado influyó sus juicios o la presencia de una indicación que lleve al auditor a considerar el efecto de la política contable de la empresa sobre la calidad de sus resultados actuales y futuros.

En síntesis, un número importante de estudios empíricos ha conseguido evidencia reveladora de la existencia de diferencias significativas entre los auditores al tomar en consideración los factores utilizados como apoyo para la toma de decisiones de materialidad. Algunos profesionales se basan primordialmente en un solo factor de referencia (el beneficio neto, por ejemplo), mientras que otros atienden a un mayor número de variables influyentes. Además, la trascendencia individual de cada factor en el proceso de formación de los juicios sobre materialidad es diferente entre los auditores.

\subsection{Diferencias en los umbrales de materialidad}

Otras investigaciones, en cambio, han identificado la utilización de diferentes niveles o umbrales cuantitativos como otra de las causas de la ausencia de consenso entre los auditores en cuanto a sus juicios sobre materialidad. Esto es, a pesar de basar sus decisiones en las mismas variables financieras, utilizan diferentes porcentajes de referencia. Resumimos en la tabla 3 las conclusiones alcanzadas por estos trabajos.

Para comenzar a analizar el conjunto de trabajos que utilizan algún criterio o atributo para categorizar a los auditores, Woolsey (1954a y b) elabora un cuestionario dirigido a profesionales que trabajan en firmas de ámbito nacional, regional y local en el que desarrolla varios casos hipotéticos para analizar si en la formación de los juicios sobre materialidad ocurre uno de los siguientes supuestos: 1) se concede mayor importancia a la cuantía absoluta de la partida o a sus importes relativos; 2) en el caso de que prevalezcan los términos relativos, qué factor o variable financiera es la base de comparación más decisiva; y 3) cuál es la línea de división, en términos absolutos o relativos, entre lo significativo y lo no significativo.

Para ello se dirige, entre otros grupos, a los auditores de cuentas estadounidenses, a los cuales clasifica en función del tipo de firma a la que pertenecen, concretando su ámbito territorial de actuación: nacional, regional y local. Los resultados de su estudio señalan que los umbrales de materialidad utilizados por los auditores al evaluar la significatividad de diferentes partidas se relacionan de forma directa con el ámbito en que sus firmas desarrollan su actividad.

De esta manera, los auditores que trabajan para firmas grandes, cuyo ámbito de actuación se enmarca dentro de todo el territorio nacional, utilizan niveles de materialidad significativamente más elevados que los aplicados por los auditores pertenecientes a firmas más pequeñas, las cuales operan únicamente dentro del ámbito local y regional. Este resultado lo consigue atendiendo a 
La materialidad en auditoría como barrera hacia la comparabilidad de la información financiera

Una revisión de la investigación empírica previa

diferentes factores cuantitativos como el beneficio antes de impuestos, el beneficio medio y el capital circulante.

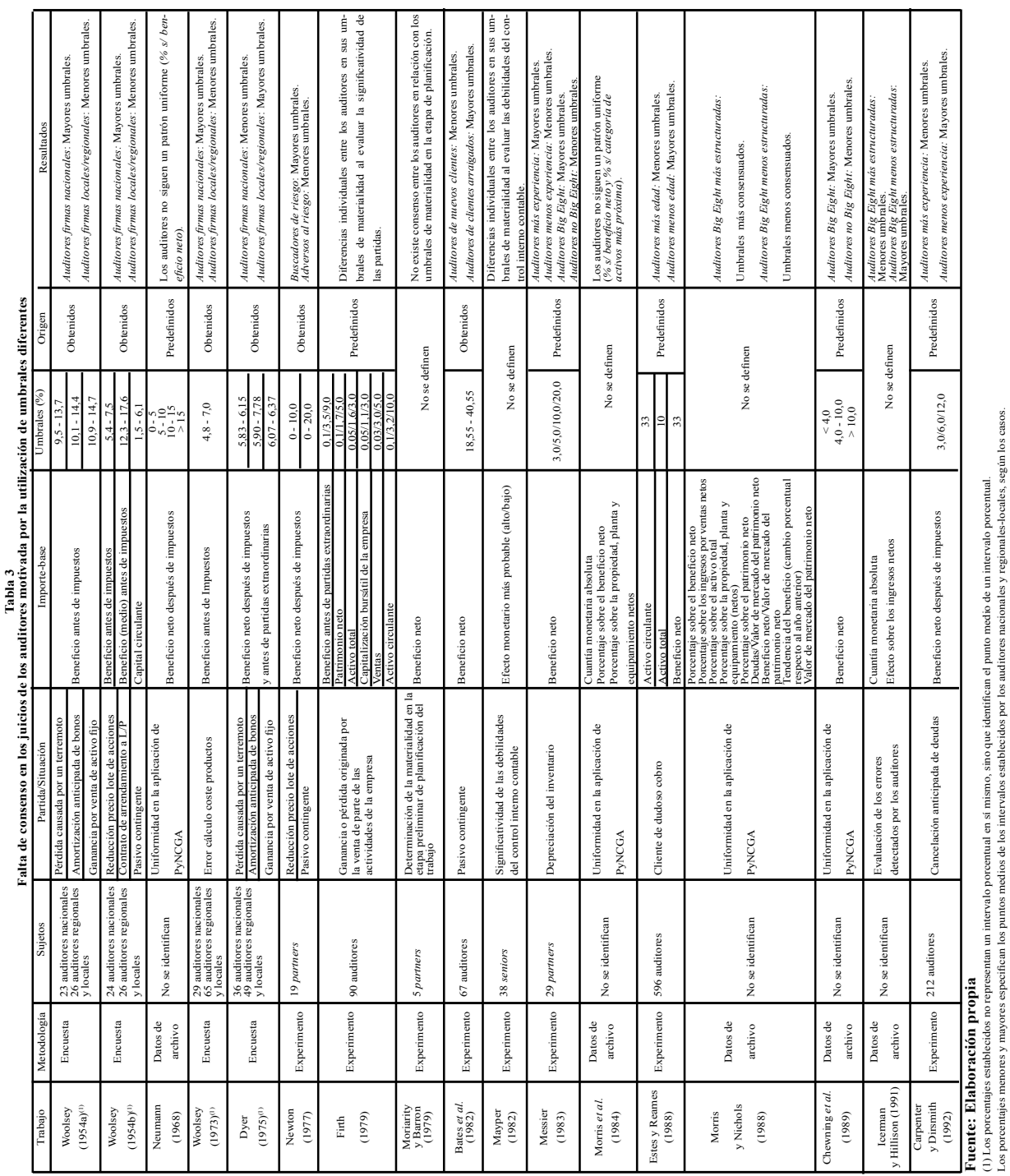

Woolsey (1954a), además, señala de forma específica que los auditores de firmas nacionales establecen la línea media de división entre partidas significativas y no significativas 4 puntos porcentuales por encima de los auditores que trabajan en el ámbito local y regional. Esta última conclusión es alcanzada por el autor utilizando como factor de referencia el beneficio antes de impuestos para tres casos concretos relacionados con 
la pérdida ocasionada por un terremoto, la amortización anticipada de unas obligaciones y la ganancia derivada de la venta de material inmovilizado.

Dos décadas más tarde, Woolsey (1973) lleva a cabo una nueva investigación con los mismos objetivos que las dos anteriores y obtiene unos resultados similares. Concretamente, para el caso de un error en la valoración de unos inventarios, los umbrales de materialidad fijados por los auditores de ámbito nacional vuelven a ser más elevados que los determinados por aquéllos integrados en firmas de menor tamaño, cuya actividad se desarrolla en el nivel regional y local. Sin embargo, puede observarse como en términos generales, utilizando como base el beneficio antes de impuestos, esta diferencia entre los límites de materialidad empleados por ambos grupos de auditores se ha recortado en el tiempo: por un lado, los umbrales de estos dos grupos son inferiores a los que establecieron veinte años antes; por otro, los umbrales utilizados por los auditores de firmas nacionales se han reducido en mayor proporción que los empleados por los que trabajan en el ámbito regional y local.

Esta reducción en los niveles de materialidad de los auditores y la merma en la diferencia entre los umbrales con que trabajan los auditores de firmas nacionales, regionales y locales es ratificada por Dyer (1975) en una réplica de la investigación de Woolsey (1954a); obtiene además, en algunos casos, unos umbrales de materialidad más elevados para el grupo integrado por los auditores de firmas regionales y locales.

Al utilizar como base la magnitud del beneficio neto después de impuestos y antes de partidas extraordinarias, los auditores de ámbito nacional siguen utilizando pautas de materialidad más flexibles que los de ámbito regional y local para el caso en que deben evaluar la significatividad de la pérdida causada por un terremoto. Por el contrario, son ahora estos últimos los que trabajan con límites de materialidad superiores para los casos de una amortización anticipada de bonos y una ganancia obtenida de la venta de material inmovilizado, aunque las diferencias entre ambos grupos son reducidas. Dyer (1975) justifica la disminución en los umbrales de materialidad utilizados por los auditores a través de los cambios producidos en el entorno, relacionados con la emisión de nueva normativa contable y con un mayor grado de concienciación por parte de los profesionales en cuanto a su responsabilidad legal.

Poco tiempo después, Newton (1977) obtiene evidencia empírica que sostiene que las decisiones de materialidad tomadas por los auditores se ven afectadas por el grado de incertidumbre que rodea la resolución final de una partida y por la disposición de éstos a aceptar el riesgo de tomar una decisión equivocada. En este estudio, los casos planteados se relacionan con una disminución continuada en la cotización de unos títulos y los daños y perjuicios derivados de la posible pérdida de un juicio. La mayoría de los individuos son clasificados en dos grupos: buscadores de riesgo y contrarios al riesgo. Los primeros generalmente utilizan umbrales de materialidad más elevados, si bien estos niveles pueden ser más estrictos si el sujeto está dispuesto a tolerar una mayor probabilidad de ocurrencia de los hechos antes de llevar una salvedad a su informe; por el contrario, 
La materialidad en auditoría como barrera hacia la comparabilidad de la información financiera

Una revisión de la investigación empírica previa

los segundos utilizan generalmente umbrales de materialidad más reducidos, aunque sus métodos para afrontar situaciones de incertidumbre pueden ser de tres tipos: 1) utilizar umbrales de materialidad relativamente rigurosos y probabilidades de ocurrencia de los hechos comparativamente estrictas (sujetos extremadamente adversos a asumir riesgos), 2) establecer umbrales de materialidad comparativamente reducidos, pero permite probabilidades de ocurrencia más moderadas y 3) emplear umbrales de materialidad aparentemente normales, pero aplicando probabilidades relativamente severas.

Al tomar como criterio la rotación del auditor responsable del trabajo de auditoría, Bates et al. (1982) diseñan un experimento a través del cual obtienen evidencia empírica acerca de que los umbrales de materialidad utilizados por los auditores para evaluar la significatividad de las partidas, de cara a su revelación en los estados financieros o bien a la emisión de un informe con opinión calificada, es función de la existencia o no de una relación previa prolongada con el cliente. Para el caso de un pasivo contingente originado por la resolución final de un litigio, cuando el auditor ha sido el encargado de auditar una compañía durante un período de tiempo extenso (cinco años), los niveles de materialidad utilizados son significativamente más elevados que los empleados cuando se produce la rotación de la firma o del socio responsable del trabajo. De este modo, aunque la importancia relativa que los auditores asignan a varios factores no se ve alterada por su grado de relación personal con el cliente, los umbrales de materialidad con los que trabajan son mayores si el profesional se ha encargado previamente de la auditoría del cliente.

Messier (1983), por otro lado, aparte de aludir a la experiencia profesional, como vimos anteriormente, usa también como criterio de clasificación el tipo de firma a la que pertenecen los auditores; así, evalúa la influencia de ambas variables en la calidad de los juicios sobre materialidad. Como resultados obtiene que el grado de consenso y la capacidad de entendimiento del proceso por seguir en la formación de estos juicios por parte de los sujetos son relativamente altos. Sin embargo, este nivel de acuerdo es superior entre los auditores con mayor experiencia y entre aquellos que trabajan dentro de alguna de las Big Eight ${ }^{4}$, que además utilizan niveles de materialidad significativamente más elevados.

En relación con el tipo de firma, el trabajo de Chewning et al. (1989) reproduce este resultado, pues a través del análisis de datos de archivo deducen la utilización de mayores niveles de materialidad para los auditores de las Big Eight cuando tienen que informar sobre la uniformidad en la aplicación de principios y normas de contabilidad generalmente aceptados (PyNCGA).

\footnotetext{
${ }^{4}$ Identificamos como Big Eight el grupo formado por las ocho grandes firmas internacionales de auditoría, integrado hasta 1989 por Arthur Andersen, Arthur Young, Coopers \& Lybrand, Deloitte Haskins \& Sells, Ernst \& Whinney, Peat Marwick Mitchel, Price Waterhouse y Touche Ross. Este colectivo se ha visto reducido a cuatro con la desaparición de Arthur Andersen en 2002 tras el escándalo financiero de Enron, por lo que actualmente es conocido como Big Four: Deloitte \& Touche, Ernst \& Young, KPMG Peat Marwick y PricewaterhouseCoopers.
} 
Respecto a la experiencia de los auditores, Carpenter y Dirsmith (1992) realizan un experimento a través del cual obtienen un resultado opuesto al conseguido previamente por Messier (1983). En este sentido, a la hora de evaluar la significatividad de tres tipos de transacciones de cancelación anticipada de deudas, los auditores con un mayor rango profesional, aquellos con experiencia previa en la revisión de dichas operaciones y los que dicen conocer la normativa pertinente trabajan con niveles de materialidad más reducidos.

Otro de los atributos que puede afectar a la formación de los juicios sobre materialidad de los auditores es su edad. Estes y Reames (1988), al respecto, obtienen evidencia empírica que demuestra la existencia de diferencias individuales significativas entre los auditores atendiendo a su edad, de tal forma que los auditores de mayor edad son más susceptibles de emitir informes con salvedades, ya que trabajan con márgenes de materialidad más estrechos. Este resultado lo obtienen para un caso hipotético en el que la partida cuya significatividad es juzgada vuelve a concretarse, como ya hiciera Messier (1983) previamente, en el nivel de obsolescencia de un inventario.

Por último, dentro de los trabajos que obtienen diferencias intergrupales en los niveles de materialidad utilizados mediante la categorización de los auditores atendiendo a distintas pautas de clasificación, encontramos dos que lo hacen observando el grado de estructuración de la firma de auditoría a la que éstos pertenecen.

En primer lugar, Morris y Nichols (1988) examinan si en la emisión de salvedades por falta de uniformidad en la aplicación de PyNCGA los umbrales de materialidad utilizados son función del nivel de estructuración de las Big Eight. Así, a pesar de encontrar diferencias significativas en el grado de consenso alcanzado entre los auditores de las diferentes Big Eight, obtienen unos resultados que evidencian un mayor acuerdo en la formación de los juicios sobre materialidad entre los auditores de las Big Eight más estructuradas. Sin concretar quiénes utilizan unos umbrales más o menos reducidos, a partir de la información suministrada por nueve variables financieras disponibles públicamente, deducen que los niveles cuantitativos empleados por los auditores de las firmas más estructuradas son significativamente más homogéneos.

Por otro lado, Icerman y Hillison (1991) disciernen entre Big Eight estructuradas, intermedias y no estructuradas; asimismo, a través de la revisión de sus papeles de trabajo, analizan la disposición de los errores detectados por sus auditores. Al respecto, afirman que las Big Eight más estructuradas son más propensas a registrar y tomar en consideración, con independencia de su cuantía, un mayor número de errores detectados, de lo cual se deduce que aplican niveles de materialidad más reducidos durante el proceso de revisión de los estados financieros.

Como indicábamos anteriormente, otra serie de investigaciones, sin embargo, han revelado una falta de uniformidad en los umbrales de materialidad utilizados por los auditores en la 
La materialidad en auditoría como barrera hacia la comparabilidad de la información financiera

Una revisión de la investigación empírica previa

formación de sus juicios sobre materialidad, sin discriminarlos conforme a determinados atributos personales o según ciertas características corporativas.

El primer caso es el trabajo de Neumann (1968) que, en relación con la formación de juicios respecto a la significatividad de los cambios en la aplicación de PyNCGA, concluye que existe una falta de consenso entre los auditores al considerar los umbrales de materialidad. Mediante el empleo del beneficio neto como pauta de referencia observa que la revelación de estos cambios por parte de las empresas, por un lado, y la calificación de la opinión en el informe de los auditores por falta de uniformidad, por otro, no siguen un patrón uniforme. En definitiva, para un importe-base comúnmente utilizado como es el beneficio neto existen diferencias significativas entre los auditores respecto a los niveles de materialidad empleados para discriminar los cambios contables con un efecto significativo sobre la imagen fiel de la empresa de aquellos otros cuyo efecto no es considerado importante.

En este mismo sentido, Morris et al. (1984) también corroboran la existencia de una falta de consenso entre los auditores. Ellos utilizan como bases el beneficio neto y la categoría de activos más relacionada con la partida evaluada; de esta forma concluyen que las opiniones calificadas por falta de uniformidad en la aplicación inicial de la capitalización de los intereses no siguen un patrón uniforme. Es decir, no existe una regla general tal que cuando el efecto de la partida sobre estos importes-base es inferior a un porcentaje $X$ ésta es considerada siempre insignificante y cuando es superior a un porcentaje $Y$ es estimada siempre significativa.

Esta misma conclusión había sido obtenida previamente por Firth (1979), quien para tres firmas diferentes deduce que sus auditores no alcanzan un grado de consenso razonable respecto a la significatividad de diversas ganancias y pérdidas extraordinarias derivadas de la venta de parte del negocio de una empresa y a la necesidad de presentarlas separadamente en los estados financieros. Aunque no se producen diferencias significativas respecto al uso y trascendencia de diferentes variables en la formación de sus juicios sobre materialidad, sí existe una notable falta de acuerdo en cuanto a la relevancia de las partidas.

El factor orientativo más importante es la partida extraordinaria expresada como porcentaje del beneficio, aunque los umbrales de materialidad utilizados por los auditores varían significativamente. Así, en muchos casos, aproximadamente la mitad de los auditores califican la partida juzgada como significativa y exigen su revelación desglosada en los estados financieros, mientras que cerca de la otra mitad no la estiman tan importante como para exigir una presentación de este tipo. Esto sugiere que los auditores no disponen de pautas cuantitativas claras o incondicionales para tomar sus decisiones de materialidad.

A los dos trabajos restantes ya nos hemos referido en el apartado previo. No obstante, ambos también revelan como causa de la falta de consenso entre los auditores la utilización de diferentes umbrales de materialidad. 
Del análisis de los resultados obtenidos por Moriarity y Barron (1979) se deriva que existen diferencias individuales en la utilización del beneficio neto como referencia para la toma de decisiones de materialidad en la etapa de planificación de la auditoría. Algunos auditores establecen unos niveles de materialidad más reducidos cuando la cifra de beneficios se aproxima a cero, mientras que otros mantienen una relación uniforme y regular entre el beneficio y la materialidad de tal forma que, a medida que el primero se reduce, los umbrales de materialidad considerados también son más reducidos.

Finalmente, destacamos la investigación de Mayper (1982), quien obtiene evidencia sobre la existencia de diferencias individuales entre los sujetos en sus umbrales de materialidad al evaluar la importancia de las debilidades en el control interno contable de las empresas.

En resumen, la falta de criterios obligatorios para la determinación de los niveles cuantitativos de materialidad lleva en muchas ocasiones a los auditores a considerar como significativos errores a importes insignificantes bajo el juicio profesional de otros auditores. Esto, junto con lo visto en el apartado anterior, influye notablemente sobre la planificación de la auditoría y el tipo de opinión emitida en el informe, lo cual repercute de forma negativa en la comparabilidad de la información financiera.

\section{Diferencias entre los auditores y otros grupos en la formación de los juicios sobre materialidad}

Como ya hemos señalado, en este apartado nos ceñimos a presentar a través de la Tabla 4 un resumen de los resultados de los trabajos que han revelado una falta de consenso entre auditores y otros grupos profesionales en la formación de los juicios sobre materialidad.

\section{Conclusiones}

A lo largo de más de medio siglo se ha llevado a cabo un importante número de investigaciones empíricas enfocadas al estudio de la materialidad en la auditoría de cuentas. Como hemos argumentado, un número considerable de estos trabajos han revelado la existencia de diferencias en la formación de los juicios sobre materialidad por parte de los auditores. Asimismo, otros han demostrado que los juicios emitidos por éstos no se corresponden con los formulados por otros grupos de individuos, como los contables, los gestores de inversiones, los analistas y ejecutivos financieros, los prestamistas o los estudiantes universitarios.

Esta falta de consenso entre los sujetos se ha puesto de manifiesto en la bibliografía previa mediante la utilización de distintas herramientas metodológicas como cuestionarios, experimentos y análisis de informaciones históricas reales. Igualmente, este desacuerdo ha sido evidenciado mediante el estudio de la significatividad de diferentes partidas (pasivos contingentes, cambios en la aplicación de PyNCGA, partidas extraordinarias, etc.) vinculadas a una multiplicidad de situaciones. 
La materialidad en auditoría como barrera hacia la comparabilidad de la información financiera Una revisión de la investigación empírica previa

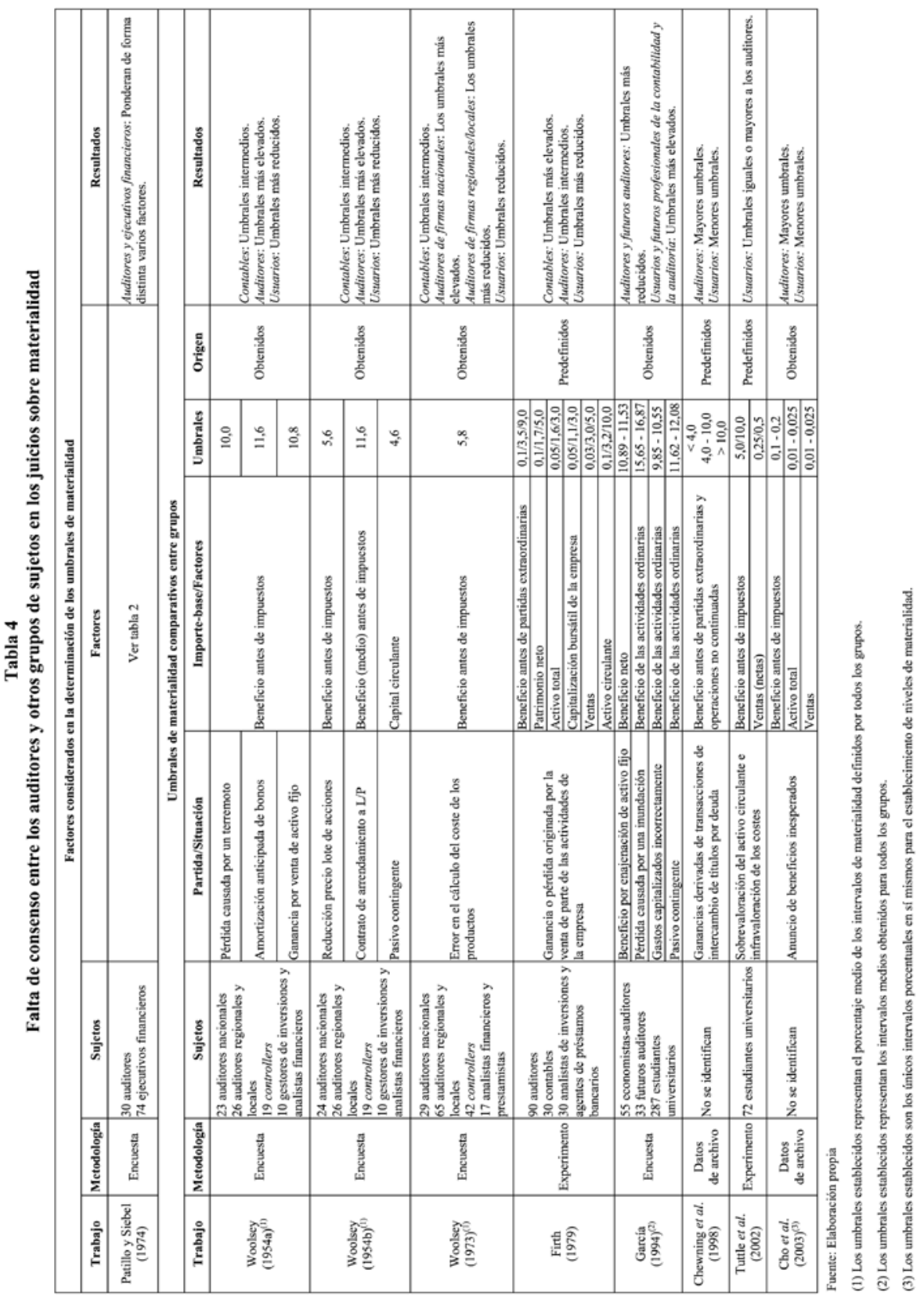

En su conjunto, estos estudios han identificado como motivos de esta discrepancia la consideración de distintos factores cuantitativos y cualitativos - tanto en naturaleza como en número-, la ponderación diferencial de la trascendencia de esos factores 
y la utilización de umbrales numéricos desiguales por parte de los individuos como referencia y apoyo para la formación de los juicios sobre materialidad.

De igual modo, ciertas características personales y corporativas relacionadas con los auditores y sus firmas han servido también para clasificar a estos sujetos en categorías e identificar diferencias intergrupales en su comportamiento ante la toma de decisiones de materialidad. Entre ellas destacan el nivel de experiencia profesional de los auditores, el tipo de firma a la que pertenecen, el enfoque de materialidad utilizado, la rotación del socio o de la firma y la propensión individual a asumir riesgos. Por lo tanto, la planificación y ejecución del trabajo, así como el tipo de opinión emitida en el informe, pueden variar significativamente en la auditoría de una determinada entidad en función de estos atributos.

En consecuencia, dentro del proceso de armonización de la información corporativa en el que nos hallamos actualmente inmersos parece necesario reconsiderar el marco teórico establecido para poder enfrentar a la vaguedad normativa que durante tantas décadas ha condicionado la aplicación del concepto de materialidad en auditoría.

En este sentido, creemos que el trabajo que desde principios del presente siglo viene realizando el IAASB para revisar la NIA 320 todavía en vigor debe erigirse a partir de ahora en el punto de referencia básico de profesionales y organismos reguladores en lo que al tema de la materialidad se refiere, ya que supone un paso muy importante hacia la consecución de unos juicios más homogéneos y uniformes por parte de los auditores, así como un mejor entendimiento por parte de los usuarios de la información financiera y de sus informes respecto a la utilización de este concepto en los trabajos de auditoría.

Para finalizar nuestro trabajo, a continuación destacamos algunos de los retos que con la entrada en vigor de la nueva normativa internacional deberán acometer los auditores con relación a la materialidad y cuya consecución debe contribuir sustancialmente a una mayor comparabilidad de la información financiera publicada por las empresas, a una mayor formalidad en la determinación de los niveles de materialidad y de las cuantías inferiores precisadas para la gestión del riesgo de la presencia de incidencias no identificadas, a una mayor atención a los cambios en las circunstancias producidos durante la auditoría, a una mayor minuciosidad en la documentación de la materialidad y en la clasificación y documentación de las incidencias detectadas, a una consideración más meticulosa de las incidencias detectadas por importes inferiores a los niveles de materialidad establecidos, así como a un mayor esfuerzo en la comunicación mantenida con los administradores y responsables del gobierno de la empresa auditada.

\section{Bibliografía}

BATES, H. L., R. W. INGRAM y P. M. J. RECKERS, (1982): “Auditor-client Affiliation: The Impact on Materiality”, en Journal of Accountancy, vol. 153, no. 4, abril, pp. 60-63.

CARPENTER, B. W. y M. W. DIRSMITH, (1992): "Early Debt Extinguishment Transactions and Auditor Materiality Judgments: A Bounded Rationality Perspective", en Accounting, Organizations and Society, vol. 17, no. 8, noviembre, pp. 709-739. 
La materialidad en auditoría como barrera hacia la comparabilidad de la información financiera

Una revisión de la investigación empírica previa

y P. P. GUPTA, (1994): "Materiality Judgments and Audit Firm Culture: Social-behavioral and Political Perspectives", en Accounting, Organizations and Society, , vol. 19, no. 4/5, mayo-julio, pp. 355-380.

CHEWNING, G., K. PANY, y S. WHEELER, (1989): “Auditor Reporting Decisions Involving Accounting Principle Changes: Some Evidence on Materiality Thresholds", en Journal of Accounting Research, vol. 27, no. 1, primavera, pp. 78-96.

CHEWNING JR., E. G., S. W. WHEELER y K. C. CHAN, (1998): "Evidence on Auditor and Investor Materiality Thresholds Resulting From equity-for-debt Swaps", en Auditing: A Journal of Practice \& Theory, vol. 17, no. 1, primavera, pp. 39-53.

CHO, S-Y. et al., (2003): "Measuring Stockholder Materiality", en Accounting Horizons, vol. 17, Supplement, pp. 63-76.

DYER, J. L., (1975): “Toward the Development of Objective Materiality Norms", en The Arthur Andersen Chronicle, vol. 35, no. 4, octubre, pp. 38-49.

ESTES, R. y D. D. REAMES, (1988): "Effect of Personal Characteristics on Materiality Decisions: A Multivariate Analysis", en Accounting and Business Research, vol. 18, no. 72, otoño, pp. 291-296.

FIRTH, M., (1979): "Consensus Views and Judgment Models in Materiality Decisions", en Accounting, Organizations and Society, vol. 4, no. 4, pp. 283-295.

GARCÍA DELGADO, S., (1994): La importancia relativa en la auditoría financiera, tesis doctoral, Universidad del País Vasco.

ICERMAN, R. C. y W. A. HILLISON, (1991): "Disposition of Audit-Detected Errors: Some Evidence on Evaluative Materiality", en Auditing: A Journal of Practice \& Theory, vol. 10, no. 1, primavera, pp. 22-34.

INTERNATIONAL ACCOUNTING STANDARDS BOARD, (1989): Framework for the preparation and presentation of financial statements, IASB, julio.

KROGSTAD, J. L., R. T. ETTENSON y J. SHANTEAU, (1984): "Context and Experience in Auditors' Materiality Judgments", en Auditing: A Journal of Practice \& Theory, vol. 4, no. 1, otoño, pp. 54-73.

MAYPER, A. G., (1982): “Consensus of Auditors' Materiality Judgments of Internal Accounting Control Weaknesses", en Journal of Accounting Research, vol. 20, no. 2 (II), otoño, pp. 773-783.

MESSIER JR., W. F., (1983): "The Effect of Experience and Firm Type on Materiality/Disclosure Judgments", en Journal of Accounting Research, vol. 21, no. 2, otoño, pp. 611-618. 
MORIARITY, S. y F. H. BARRON, (1976): "Modeling the Materiality Judgments of Audit Partners”, en Journal of Accounting Research, vol. 14, no. 2, otoño, pp. 320-341.

(1979): “A Judgment-Based Definition of Materiality”, en Journal of Accounting Research, vol. 17, Supplement, pp. 114-135.

MORRIS, M. H., W. D. NICHOLS y J. W. PATILLO, (1984): “Capitalization of Interest, Materiality Judgment Divergence, and Users' Information Needs", en Journal of Business, Finance and Accounting, vol. 11, no. 4, diciembre, pp. 547-555.

MORRIS, M. H. y W. D. NICHOLS, (1988): “Consistency Exceptions: Materiality Judgments and Audit Firm Structure”, en The Accounting Review, vol. 63, no. 2, abril, pp. 237-254.

NELSON, M. W., S. D. SMITH y Z.-V. PALMROSE, (2005): “The Effect of Quantitative Materiality Approach on Auditors' Adjustment Decisions", en The Accounting Review, vol. 80, no. 3, julio, pp. 897-920.

NEUMANN, F., (1968): “The Auditing Standard of Consistency”, en Journal of Accounting Research, vol. 6, Supplement, pp. 1-17.

NEWTON, L. K., (1977): “The Risk Factor in Materiality Decisions", en The Accounting Review, vol. 52, no. 1, enero, pp. 97-108.

PATILLO, J. W. y J. D. SIEBEL, (1974): "Factors Affecting the Materiality Judgment", en The CPA Journal, vol. 44, no. 7, julio, pp. 39-44.

SIERRA MOLINA, G. y M. G. SANTA MARÍA PÉREZ, M. G. (2002): “La formación del juicio profesional: las diferencias individuales del auditor", en Revista de Contabilidad, vol. 5, no. 10, julio-diciembre, pp. 179-203.

TUTTLE, B., M. COLLER y R. D. PLUMLEE, (2002): “The Effect of Misstatements on Decision of Financial Statements Users: An Experimental Investigation of Auditor Materiality Thresholds", en Auditing: A Journal of Practice \& Theory, vol. 21, no. 1, marzo, pp. 11-27.

WOOLSEY, S. M., (1954a): "Development of Criteria to Guide the Accountant in Judging Materiality”, en Journal of Accountancy, vol. 97, no. 2, febrero, pp. 167-173.

Disclosure", en Journal of Accountancy, vol. 98, no. 6, diciembre, pp. 745-750. 3, septiembre, pp. 91-92. (A)

, (1973): “Materiality Survey", en Journal of Accountancy, vol. 136, no. 